\title{
Heat Artifact Mimicking Adenocarcinoma of Fallopian Tube
}

\author{
Prajakta Bapat*, Sujaya Mazumder and Susan Cherian
}

Department of Pathology, BARC Hospital, Mumbai. 400094

\section{Dear Sir,}

A 45 years old female presented to the gynecology outpatient department with history of pain in the abdomen and irregular menstrual cycles. Radiological examination revealed a right ovarian mass of size $3.5 \times 3 \times 1 \mathrm{~cm}$ and uterus showed multiple tiny fibroids. Left ovary and bilateral tubes were normal on radiology. Remaining medical history was non- contributory. Patient underwent total abdominal hysterectomy with bilateral salphingo-opherectomy and we received the specimen for histopathology. On gross examination, the uterus showed multiple intramural fibroids ranging in size from 3 to $1 \mathrm{~cm}$. Right ovary showed a simple cyst measuring $3.2 \times 2.5 \times 0.6 \mathrm{~cm}$ having maximum wall thickness $0.3 \mathrm{~cm}$ which yielded serous fluid. Both the tubes and the left ovary were grossly unremarkable. No mass, thickening or dilatation of tubes observed. On histopathological examination, the right ovarian mass was a benign simple serous cystadenoma and the right fallopian tubes also showed normal histology. Left ovary was within normal limits. However, left fallopian tube showed pseudostratification of the luminal layer which was lined by tall, elongated, hyperchromatic nuclei exhibiting mild nuclear atypia.(figure 1,2). There was no mitosis or loss of polarity. At places it showed a papillaroid pattern and apparent proliferation of the epithelium (figure 3). All these findings initially lead to the doubt of a malignancy amidst the residents. After careful re-examination of the specimen, review of the slides by senior pathologist and history of use of thermal cautery intraoperatively by the gynecologists, the findings were finally attributed to heat induced artefactual changes.

Artefact is defined as an artificial structure or tissue alteration on a microscopic slide as a result of extraneous factor. ${ }^{[1]}$ Artefacts can be a major source of diagnostic challenge in day to day practice of a pathologist and it is important to recognize them to avoid misdiagnosis. One such commonly encountered artefact is the heat/fulguration artifact. ${ }^{[2]}$ Heat produced while electrosurgical procedures or while using laser intraoperatively can microscopically produce marked changes in the form of mild to moderate nuclear atypia with features such as nuclear elongation, stratification, hyperchromasia and smudging with obliteration of cell boundaries. ${ }^{[3,4]}$ These features along with papillaroid pattern of the tubal epithelium are the principle features which can lead to the doubt of dysplasia or in situ malignancy as in our case. The papillary folds are possibly due to protrusion of partially detached segments of the tubal epithelium. The epithelium gets detached due to the pressure by water vapor produced by the heat along with heat induced swelling of the tubal villi. ${ }^{[4]}$

Though primary fallopian tube malignancy is rare, it has a high mortality rate and also are now linked with the pathogenesis of serous epithelial tumors of the ovary. ${ }^{[5]}$ This has led to the current practice of extensive sampling of the fallopian tube as compared to before by Sectioning and Extensively Examining the FIMbriated End (SEEFIM) protocol. ${ }^{[6]}$ With the increase in sampling of fallopian tube specimen, it may be common to encounter artefactual changes caused by heat from cautery used in surgery and may cause confusion among young budding pathologists. Therefore, it is important to keep in mind the changes in fallopian tube morphology that is caused by heat to avoid any overdiagnosis of malignancy.

Heatchanges can be distinguished fromserous intraepithelial carcinoma (STIC) by absence of mitosis, basophilia of the surrounding connective tissue and differences in nuclear morphology. ${ }^{[3]}$ Separation of the tubal epithelium from the core is another key feature of heat artefactual change. If further doubt prevails, immunohistochemistry can demonstrate aberrant p53 protein expression (diffuse nuclear overexpression or complete absence of staining) and an increased $\mathrm{Ki}-67$ proliferation index in a STIC lesion. ${ }^{[7]}$ Another differential diagnosis to consider would be a benign papilloma. Papillomas grossly presents as obstructive lesions and microscopically papillae have a complex branching pattern with loose fibrovascular cores. ${ }^{[8]}$ These features can help us differentiate it from the heat artefactual change.

In conclusion, heat artefactual change in a fallopian tube can microscopically mimic adenocarcinoma or even benign papillary lesion of the fallopian tube. This article aims to create awareness of this potential source of diagnostic dilemma. 


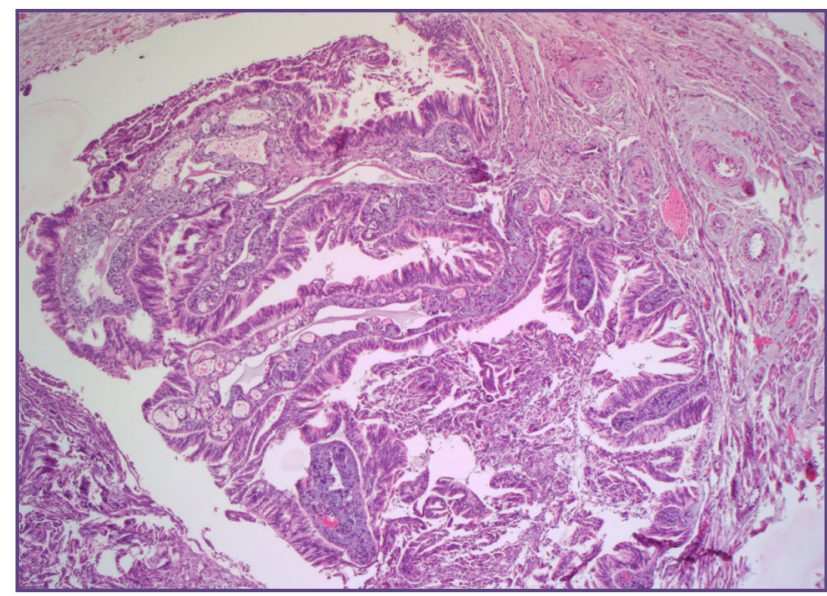

Fig.1: Photomicrograph of sections from the left fallopian tube showing, luminal layer lined by tall columnar cells with elongated hyperchromatic nuclei (H\&E 50x).

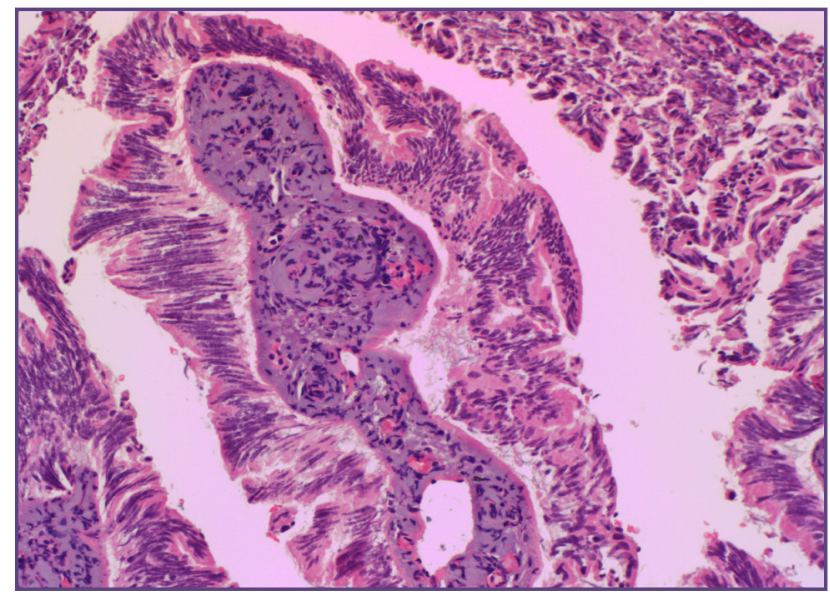

Fig. 2: Photomicrograph of sections from the left fallopian tube showing pseudostratification and mild nuclear atypia (H\&E 200x).

\section{Funding}

Nil

\section{Competing Interests}

No

\section{Reference}

1. Seoane J, Varela-Centelles PI, Ramirez JR, CameselleTeijeiro J, Romero MA. Artefacts in oral incisional biopsies

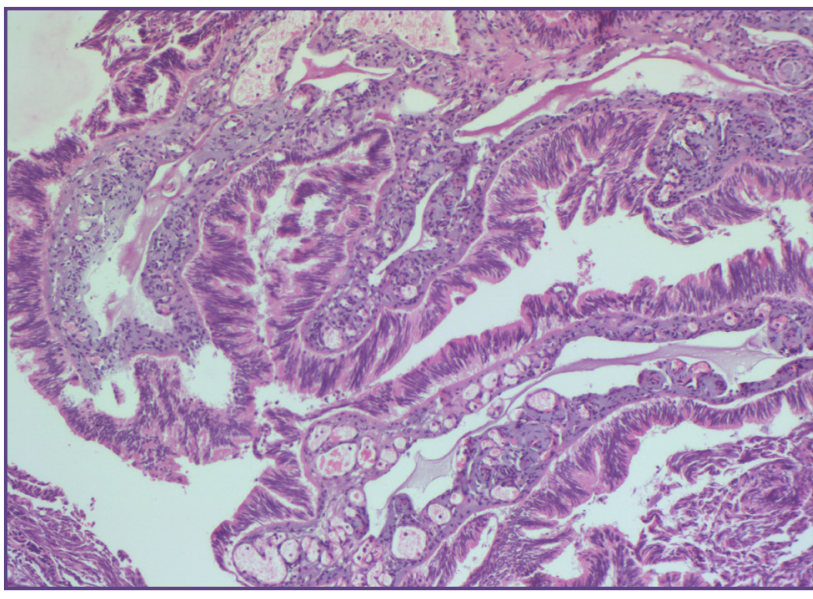

Fig.3: Photomicrograph of sections from the left fallopian tube showing papillaroid pattern with apparent epithelial proliferation (H\&E 100x).

in general dental practice: A pathology audit.Oral Dis 2004;10:113-7.

2. 2.Chatterjee S. Artefacts in histopathology. J Oral Maxillofac Pathol 2014;18:111-6.

3. Thor AD, Young RH, Clement PB. Pathology of the fallopian tube, broad ligament, peritoneum, and pelvic soft tissues. Hum Pathol 1991;22:856-67.

4. Cornog JL, Currie JL, Rubin A.Heat artifact simulating adenocarcinoma of fallopian tube. JAMA 1970;214:1118-9

5. Hyriavenko N, Lyndin M, Sikora $K$ et al.Serous Adenocarcinoma of Fallopian Tubes:Histological and Immunohistochemical Aspects. $\mathrm{J}$ pathol transl med 2019;534:236-43.

6. Arora S, Yelikar BR, Karigoudar MH. Evaluation of SEEFIM (Sectioning and Extensively Examining the FIMbriated End) Protocol in Identifying Fallopian Tube Precursor Lesions in Women with Ovarian Tumors. J Obstet Gynecol India 2019;69:153-9.

7. Visvanathan K, Vang R, Shaw P, et al. Diagnosis of serous tubal intraepithelial carcinoma based on morphologic and immunohistochemical features: a reproducibility study. Am J Surg Pathol 2011;35:1766-1775.

8. Rathore R, Singh U, Sharma S. Fallopian Tube Papilloma: An Unusual Case of Tubal Obstruction. J Clin Gynaecol Obstet 2016;2:71-3.

\section{*Corresponding author:}

Bapat Prajakta M, PG Student, Department of Pathology, BARC Hospital Anushaktinagar, Mumbai. 400094

Phone: +91 7620122166

Email: drprajaktack@gmail.com

Financial or other Competing Interests: None.

Date of Submission : 30/06/2020

Date of Acceptance : 06/09/2020

Date of Publication : 30/10/2020 